\title{
CONCEPÇÕES DE AMBIENTE DE FUTURAS PROFESSORAS PORTUGUESAS: UM ESTUDO DE CASO
}

\author{
Marisa Monteiro Correia ${ }^{1}$
}

\begin{abstract}
Resumo: Nos últimos anos, a investigação realizada na área da educação ambiental tem apontado as concepções de ambiente de alunos e de professores como um dos maiores obstáculos à consecução dos objetivos da educação ambiental no sistema educativo formal. Este artigo relata um estudo que teve como objetivo analisar as concepções de ambiente de um grupo de 32 estudantes que frequentavam um curso de formação de professores numa instituição de ensino superior portuguesa. A recolha de dados consistiu na resposta a uma questão aberta sobre o que os participantes entendiam por ambiente. Os resultados indicam que, maioritariamente, as estudantes apresentam concepções que refletem a ideia de ambiente como um objeto e não como a relação entre o mundo natural e os sistemas sociais, como tem sido defendido na literatura.
\end{abstract}

Palavras-chave: Concepções de professores. Ambiente. Educação Ambiental.

\section{PORTUGUESE PRE-SERVICE TEACHERS CONCEPTIONS ABOUT THE ENVIRONMENT: A CASE STUDY}

\begin{abstract}
In the last years, research conducted in the field of environmental education has pointed students' and teachers' conceptions of environment as one of the biggest obstacles to achieving the goals of environmental education in the formal education system. This article reports a study that aimed to examine the conceptions about the environment of a group of 32 students attending a training course for teachers and educators in a portuguese institution of higher learning. Data collection consisted in response to an open question about what participants understand by environment. The results indicate that mostly the students present concepts that reflect the idea of the environment as an object and not as the relationship between the natural and social systems, as has been argued in the literature.
\end{abstract}

Keywords: Teachers' Conceptions. Environment. Environmental Education.

\section{CONCEPCIONES DE AMBIENTE DE FUTURAS PROFESORAS PORTUGUESAS: UN ESTUDIO DE CASO.}

Resumen: En los últimos años, la investigación realizada en el área de educación ambiental ha apuntado las concepciones de ambiente de alumnos y de profesores como uno de los mayores obstáculos para la consecución de los objetivos de la educación ambiental en el

\footnotetext{
${ }^{1}$ Licenciada em Ensino da Química e da Física, Mestre em Educação, Professora na Escola Superior de Educação de Santarém (Departamento de Ciências Matemáticas e Naturais), Doutoranda em Educação no Instituto de educação da Universidade de Lisboa (ULISBOA), Santarém, Portugal, marisa.correia@ese.ipsantarem.pt
} 
sistema educativo formal. Este artículo relata un estudio que tuvo como objetivo analizar las concepciones de ambiente de un grupo de 32 estudiantes que frecuentaban un curso de formación de profesores en una institución de enseñanza superior portuguesa. La recogida de datos consistió en la respuesta a una pregunta abierta sobre lo que los participantes entendían por ambiente. Los resultados indican que, mayoritariamente, las estudiantes presentan concepciones que reflejan la idea de ambiente como un objeto y no como la relación entre el mundo natural y los sistemas sociales, como se ha defendido en la literatura.

Palabras clave: Concepciones de profesores. Ambiente. Educación Ambiental.

\section{Introdução}

Há mais de 30 anos que a UNESCO apelou à inclusão da Educação Ambiental nos currículos escolares, como forma de fazer face a "repercussões nefastas sobre a sociedade e o ambiente" (UNESCO-UNEP, 1976) provocadas por um crescimento económico e tecnológico sem precedentes. Apesar do contributo das conferências da ONU e do interesse cada vez maior que as problemáticas ambientais têm suscitado à classe política, a promoção de uma educação ambiental formal não tem sido bem-sucedida (GIORDAN, 2001). Esta situação deve-se a diversos fatores, como por exemplo, a ideia de que os temas relacionados com o ambiente são abordados prioritariamente em determinadas disciplinas escolares (Biologia, Geologia e Geografia) (ALMEIDA; VASCONCELOS, 2013; PALMER, 1998; WALSCHE, 2008). Parlo e Butler (2007) mencionam a falta de formação dos professores como um impedimento à implementação da educação ambiental em sala de aula. Urda (2004) enfatiza, ainda, outra dificuldade na abordagem da educação ambiental na educação formal relacionada com a existência de diferentes significados para o termo ambiente na nossa sociedade.

Em Portugal, as preocupações ambientais começaram a surgir após a revolução de 1974. Desde aí, têm-se multiplicado o número de iniciativas desenvolvidas na escolas com um caráter continuado, integrado e contextualizado (BAPTISTA; VILARIGUES, 2001). Contudo, de acordo com Almeida (2007) os projetos desenvolvidos nas escolas focam-se na temática da política dos 3Rs, o que reflete uma concepção da educação ambiental centrada na gestão dos recursos. Esta concepção, como salienta o autor, está associada a uma perspetiva antropocêntrica da relação Homem-Natureza, que é responsável pela crise ambiental que vivemos. Segundo Urda (2004, p.1), a concepção de ambiente dos indivíduos influencia diretamente a educação ambiental, tratando-se de uma interação entre os sistemas naturais e os sistemas sociais: "as sociedades utilizam recursos e intervêm sobre a natureza e, ao mesmo tempo, as condições naturais do mundo, fornecem e respondem às intervenções humanas". Assim, identificar as concepções de ambiente de professores é essencial não só para compreender a sua influência sobre as práticas pedagógicas, como defendido por Almeida (2007), mas, também, para conceber estratégias de mudança dessas concepções no âmbito da formação inicial e contínua de professores.

$\mathrm{Na}$ literatura são várias as perspetivas ambientalistas apresentadas - antroponcêntrica, biocêntrica e ecocêntrica. As relações entre o Homem (anthropos) e o Universo estão na base do antropocentrismo (MÁXIMO-ESTEVES, 1998) que "assume a centralidade indiscutível do ser humano e valoriza a natureza partindo de uma perspetiva instrumental" (ALMEIDA; VASCONCELOS, 2013, p. 3). Do ponto de vista antropocêntrico a natureza e a biodiversidade têm que ser preservadas, caso contrário seria prejudicial para a humanidade (OKSANEN, 1997). Almeida (2007) descreve duas abordagens ao antropocentrismo, por vezes irreconciliáveis. A primeira, de teor economicista, entende a natureza como um recurso. A segunda, relacionada 
com outros interesses "que se prendem com o usufruto da natureza e que são indissociáveis do papel que esta parece desempenhar no desenvolvimento integral do ser humano em termos psicossomáticos" (ALMEIDA, 2007, p. 30). No que se refere à perspetiva biocêntrica, esta rompe com a ideia do valor instrumental da natureza, defendendo o valor intrínseco das outras formas de vida sem considerar a sua relevância para a espécie humana (ALMEIDA, 2007; ALMEIDA; VASCONCELOS, 2013; LOCKWOOD, 1999; MÁXIMO-ESTEVES, 1998; OKSANEN, 1997). Paul Taylor (1989) defende uma forma de biocentrismo designado por igualitarismo biológico, assente no princípio que todos os seres vivos são iguais. Outros autores (por exemplo, SINGER, 1990) defendem um biocentrismo mais moderado ao atribuírem um valor superior a espécies capazes de sentir prazer ou sofrimento. A perspetiva ecocêntrica referese a uma concepção mais abrangente, que, para além de incluir as formas de vida, também inclui os ecossistemas, a biosfera ou os processos que mantêm essas entidades LOCKWOOD, 1999; OKSANEN, 1997). Como destaca Almeida (2007), o equilíbrio dos ecossistemas depende também de outros elementos, tais como o solo, a água, os processos biológicos, físico-químicos e geológicos, que ocorrem nos ecossistemas.

As perspetivas ambientalistas são classificadas frequentemente de acordo com a sua posição num continum entre dois polos - antropocêntrico e ecocêntrico (THOMPSON; BARTON, 1994; BJERKE; KALTENBORN, 1999). Alguns autores (WISEMAN; BOGNER, 2003; MILFONT; DUCKITT, 2006) optam pela terminologia utilização e preservação a que fazem corresponder as duas perspetivas opostas. Outros sugeriram que as orientações acerca do ambiente podem variar de tecnocráticas a ecocêntricas. A este respeito, Killingsworth e Palmer (1992) apresentam um continum das perspetivas de ambiente, com a natureza como objeto num dos extremos, a natureza como um recurso numa posição intermédia e, no polo oposto, a natureza como um espírito. Já Stem, Dietz e Kalof (1993) apresentam três orientações: egoísta, socialmente altruísta e biosférica.

A preocupação com as concepções de ambiente dos indivíduos tem conduzido a um crescimento considerável de investigação nessa área. Dunlap et al. (2000) desenvolveram um instrumento New Environmental Paradigm (NEP) Scale com quinze itens para medir as crenças, as atitudes e os valores ambientais. Thompson e Barton (1994) conceberam uma escala em que acrescentaram às orientações ecocêntrica e antropocêntrica uma terceira dimensão para medir a apatia ambiental. O trabalho desenvolvido por Tracana, Ferreira e Carvalho (2012) revelou que os professores e futuros professores primários (do $1^{\circ}$ ciclo do ensino básico) e de Biologia, que são os que mais implementam a educação ambiental, aproximam-se mais de uma perspetiva ecocêntrica do que os professores de Língua Portuguesa, que defendem uma posição tendencialmente mais antropocêntrica. Os estudos realizados por Almeida (2007) e Almeida e Vasconcelos (2013) envolveram sessenta professores de quatro ciclos de ensino (pré-escolar, $1^{\circ}$ ciclo, $2^{\circ}$ ciclo, $3^{\circ}$ ciclo e secundário). A análise das entrevistas evidenciou uma elevada ocorrência de perspetivas biocêntricas, em particular nos professores generalistas. Os professores de níveis superiores de ensino demonstraram mais percepções ecocêntricas. De acordo com os autores, essa situação poderá dever-se aos modelos de formação inicial e contínua dos professores e às características especificas do nível de ensino em questão. Por outro lado, os projetos ambientais desenvolvidos pelos professores centram-se na sustentabilidade no uso dos recursos, o que está em conformidade com argumentos antropocêntricos. Esses resultados permitem concluir que é possível a coexistência de diferentes perspetivas ambientalistas nos professores. A investigação levada a cabo por Kahn e Lourenço (2002) pretendia identificar a argumentação antropocêntrica ou biocêntrica de crianças e adolescentes quando questionados acerca de problemáticas ambientais. Este estudo realizado em três regiões - Houston (EUA), Amazónia (Brazil) e Lisboa (Portugal), revelou uma prevalência na argumentação antropocêntrica e que a argumentação biocêntrica tende a aumentar progressivamente com a idade dos alunos. À semelhança dos estudos referidos anteriormente, também este último conclui que na idade adulta os indivíduos podem possuir mais do que uma perspetiva ambientalista. 
Inúmeros estudos têm sido desenvolvidos com crianças e jovens com o objetivo de identificar as suas concepções de ambiente (ALERBY, 2000; ALMEIDA et al., 2011; BARRAZA, 1999; KALVAITIS; MONHARDT, 2012; LOUGHLAND; REID; PETOCZ, 2002; SHEPARDSON, 2005; SHEPARDSON et al., 2007). Alguns desses estudos recorrem à análise de desenhos elaborados pelas crianças (ALERBY, 2000; BARRAZA, 1999; KALVAITIS; MONHARDT, 2012; SHEPARDSON, 2005; SHEPARDSON et al., 2007), narrativas (KALVAITIS; MONHARDT, 2012; SHEPARDSON, 2005; SHEPARDSON et al., 2007) e fotografias (SHEPARDSON, 2005; SHEPARDSON et al., 2007).

Shepardson (2005) constatou, no seu estudo com 81 alunos do ensino médio nos Estados Unidos da América, que o ambiente foi associado a uma paisagem natural e que as paisagens alteradas pelo homem não foram consideradas parte integrante do ambiente. Para esses alunos, os humanos estão à parte do ambiente. Noutro estudo (SHEPARDSON et al., 2007) envolvendo escolas do ensino primário até ao ensino secundário, da análise das respostas dos alunos emergiram quatro modelos mentais: Modelo 1, o ambiente como um lugar onde os animais e as plantas vivem; Modelo 2, o ambiente como um lugar que sustenta a vida; Modelo 3, o ambiente como um lugar modificado pela atividade humana; Modelo 4, o ambiente como um lugar em que os animais, as plantas e os humanos vivem. Os autores verificaram que o modelo dominante era o primeiro, seguido do terceiro.

Loughland, Reid e Petocz (2002, p.187) estudaram, na Austrália, as respostas de cerca de 2000 alunos do ensino primário e secundário à seguinte questão: "Eu penso que o termo/a palavra ambiente significa...". Eles identificaram seis conceptualizações de ambiente distintas: o ambiente como um lugar; o ambiente como um lugar que contém formas de vida; o ambiente como um lugar que contém formas de vida e pessoas; o ambiente que faz coisas pelas pessoas; as pessoas são parte do ambiente e são responsáveis por ele; e as pessoas e o ambiente interagem entre si. As primeiras três categorias, segundo os autores, refletem a ideia do ambiente como um objeto e as últimas três refletem a ideia de que as pessoas e o ambiente estão relacionados. Essas concepções foram organizadas de forma hierárquica, partindo de um entendimento do termo mais limitado para uma visão mais inclusiva e abrangente. Os resultados apontaram para o predomínio de uma concepção de ambiente como um lugar, possivelmente incluindo plantas e animais, mas essencialmente separado das pessoas, em conformidade com a visão do ambiente como um objeto. Constatou-se, ainda, que a frequência da concepção de ambiente como uma relação vai decaindo à medida que aumenta o nível de escolaridade.

Os mesmos autores desenvolveram outro estudo, que incidiu nas concepções de ambiente de adultos (PETOCZ; REID; LOUGHLAND, 2003, p.2). Foram analisadas 436 respostas à seguinte questão: "O que significa ambiente para si pessoalmente?" Os resultados são semelhantes aos do estudo anterior, o que indica que as concepções das pessoas, no essencial, não sofrem alterações com a maturidade. Apesar de reconhecerem que os estudantes universitários constituem um grupo importante para a educação ambiental, uma vez que formarão a próxima geração de profissionais, os autores não consideraram o ensino superior nas investigações que realizaram. Urda (2004, p.5), tendo por base as conceptualizações desses autores, estudou as concepções de estudantes do ensino superior que frequentavam cursos na área do ambiente. Os resultados obtidos revelaram que, quer quando ingressam nos cursos como um ano depois, as ideias dos estudantes acerca do ambiente mantêm-se "maioritariamente centradas na ideia de um lugar ou sítio físico. Os aspetos relacionais ou de interação aparecem na minoria das respostas". Apenas uma resposta considerou a relação entre os sistemas sociais e os sistemas naturais.

Assim, neste trabalho considerou-se pertinente conhecer as concepções de ambiente de futuros professores, porque, como destaca Knapp (2000), estes têm um papel fundamental para o desenvolvimento da literacia ambiental e formação de cidadãos responsáveis, a quem caberá tomar decisões de forma a resolver os problemas ambientais. 


\section{Metodologia do Estudo}

Neste trabalho optou-se por uma investigação qualitativa de orientação interpretativa, em que alguns dados podem ser quantificados, mas a maioria da análise é interpretativa (STRAUSS; CORBIN, 1998). Recorreu-se à metodologia de estudo de caso para explorar as perspetivas ambientalistas de um grupo de estudantes de uma instituição de ensino superior. Esse tipo de metodologia é útil para compreender os fenómenos educativos de uma forma holística e rica (MERRIAM, 2009) e é a mais adequada quando se pretende investigar "um fenómeno no seu ambiente natural, quando as fronteiras entre o fenómeno e o contexto não são bem definidas" (YIN, 2003 p. 13). O estudo de caso é, assim, um estudo detalhado que pode ser conduzido com o propósito de explorar, descrever ou explicar um determinado fenómeno em profundidade (YIN, 2003).

Este artigo constitui um extrato de estudo mais amplo sobre a mudança de concepções de futuros professores acerca do ambiente e da educação ambiental após a frequência de uma disciplina de Educação Ambiental no âmbito da licenciatura. Participaram no estudo uma turma do Curso de Educação Básica do $3^{\circ}$ ano da licenciatura da Escola Superior de Educação, pertencente ao Instituto Politécnico de Santarém. A turma era composta por trinta e dois estudantes do sexo feminino que no final da licenciatura se candidatam aos cursos de mestrado que os habilitam para a docência no Pré-Escolar, no $1^{\circ}$ Ciclo ou no $2^{\circ}$ Ciclo. As estudantes aquando da participação neste estudo não tinham frequentado disciplinas na área da educação ambiental no seu curso. As participantes no estudo são denominadas por letras numeradas, de E1 a E32. A maioria das estudantes teve um percurso escolar anterior ao ensino superior na área das ciências sociais e humanas. Não constitui foco deste estudo analisar a influência do género, da idade e do percurso académico nas perspetivas dos estudantes. Contudo, esses aspetos serão alvo de análise futura.

A recolha de dados consistiu na resposta por escrito a uma questão aberta: Quando pensa em ambiente, o que lhe vem à mente?. Este método de recolha de dados, que Patton (2002) define como documentos escritos pelos professores, possibilita "férteis descrições de como as pessoas que produziram os materiais pensam acerca do seu mundo" (BOGDAN; BIKLEN, 1994, p. 176). De acordo com Merriam (2009), esses documentos pessoais são produzidos pelos participantes e, por isso, são credíveis relativamente às atitudes, às concepções e à visão do sujeito sobre o mundo que o rodeia.

No processo de análise de dados emergiu um quadro categorial, cujas categorias foram pré-definidas tendo em consideração as classificação das concepções de ambiente propostas por Loughland, Reid e Petocz (2002) e por Urda (2004). Assim, consideraram-se duas categorias: concepções de ambiente focadas no objeto e concepções de ambiente focadas na relação. As categorias e subcategorias sistematizam-se no Quadro 1.

Quadro 1 - Categorias e Subcategorias de análise Respeitantes às Concepções de Ambiente

\begin{tabular}{|c|c|}
\hline Categoria & Subcategoria \\
\hline \multirow{3}{*}{ Concepções centradas no objeto } & O ambiente é um lugar \\
\hline & O ambiente é um lugar que contém coisas vivas \\
\hline & O ambiente é um lugar que contém coisas vivas e pessoas \\
\hline \multirow{3}{*}{ Concepções centradas na relação } & O ambiente faz coisas pelas pessoas \\
\hline & As pessoas fazem parte do ambiente e são responsáveis por ele \\
\hline & $\begin{array}{l}\text { O ambiente é o resultado da interação entre os sistemas sociais e os } \\
\text { sistemas naturais }\end{array}$ \\
\hline
\end{tabular}

Fonte: a autora 


\section{Resultados}

A análise das respostas das estudantes permitiu constatar que a maioria apresentou concepções de ambiente centradas no objeto, como ilustra a Figura 1. Resultados semelhantes aos obtidos nos estudos realizados por Loughland, Reid e Petocz (2002) e por Urda (2004). No entanto, as percentagens obtidas nesta investigação, relativamente às concepções centradas na relação são claramente superiores quando comparadas com os resultados obtidos nos estudos mencionados. A Figura 2 apresenta as percentagens de estudantes que evidenciaram cada uma das concepções de ambiente estudadas.

Figura 1 - Diferentes Enfoques das Concepções de Ambiente

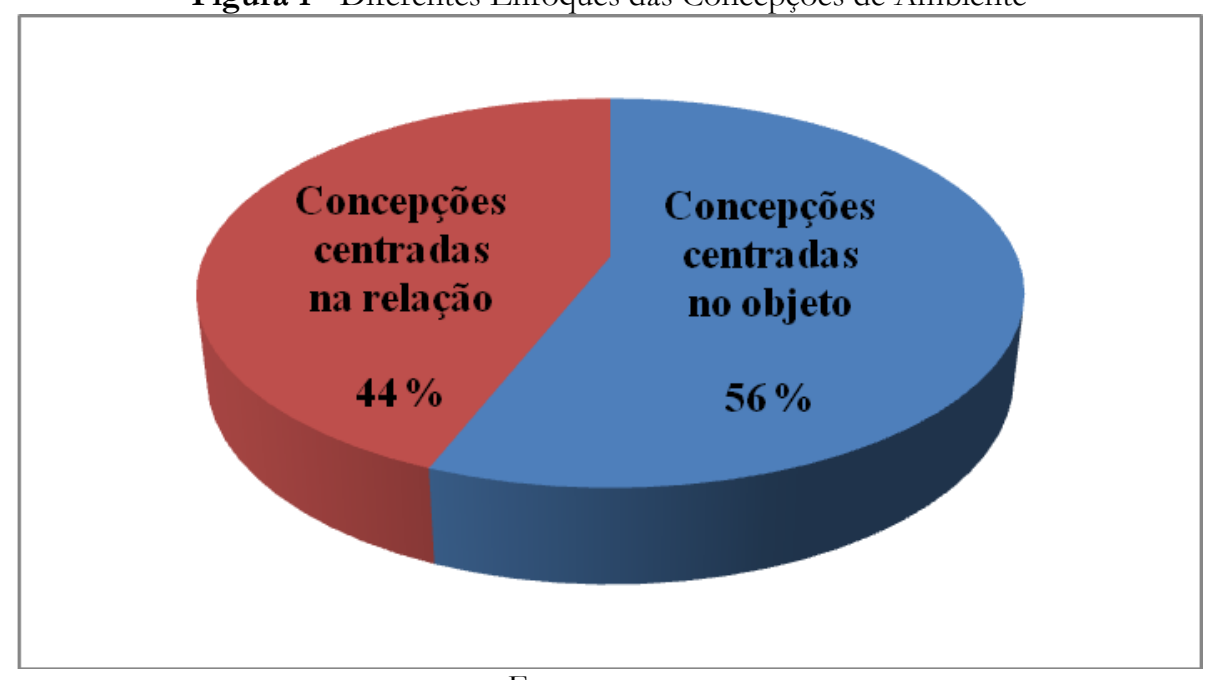

Fonte: a autora

Figura 2 - Concepções de Ambiente

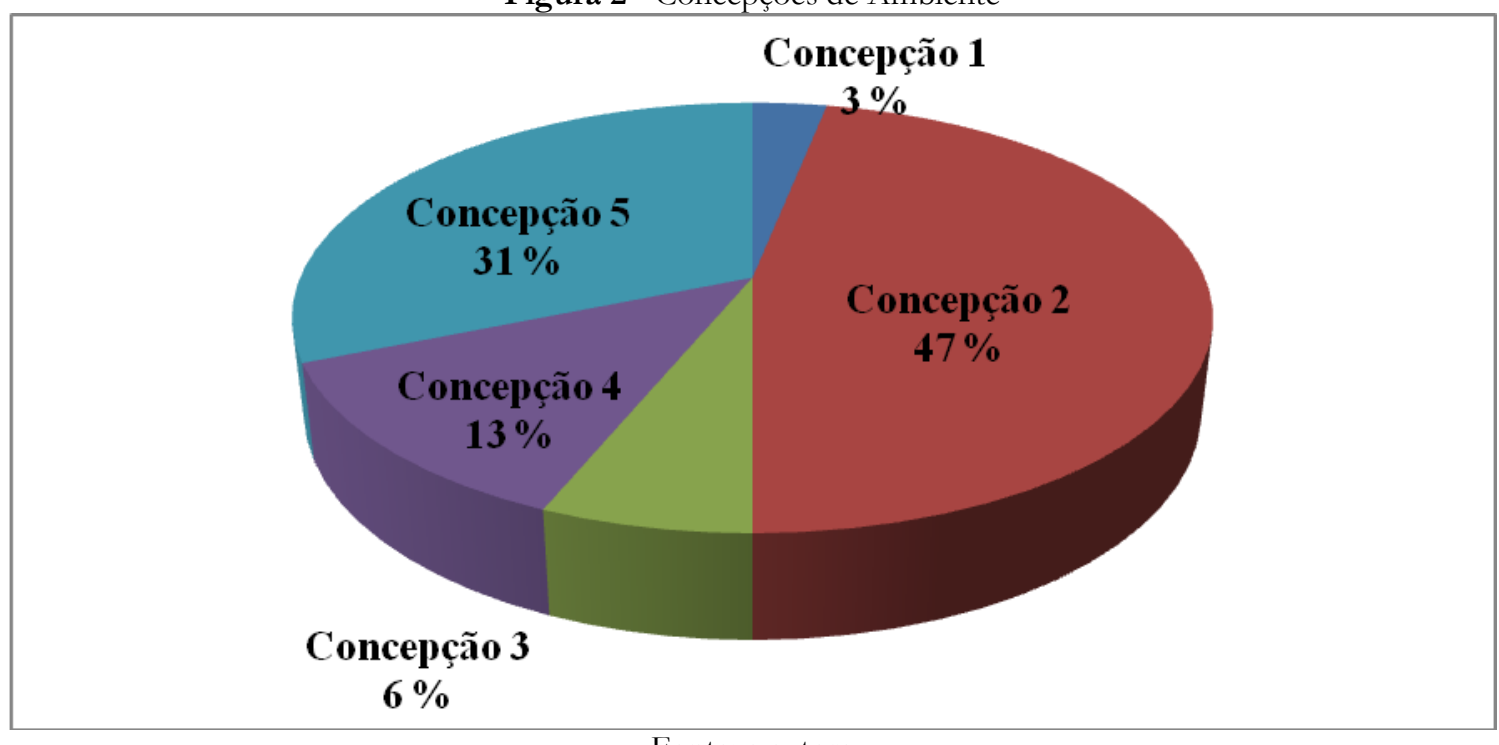

Fonte: a autora 
Uma grande parte das futuras professoras inquiridas apresentam a ideia de que "ambiente remete para tudo o que está relacionado com a natureza, basicamente, quase tudo o que nos rodeia" (E32). Subjacente a esta afirmação está a concepção de que ambiente é um lugar que contém coisas vivas (Concepção 2). Seis dessas estudantes consideram mesmo que ambiente é sinónimo de natureza. Uma estudante revelou uma visão simplista (Concepção 1), considerando apenas que "o ambiente é o espaço que nos envolve!" (E28). Apenas duas estudantes combinam essas duas concepções acrescentando outra componente, as pessoas (Concepção 3). Como se depreende da seguinte afirmação: "Quando penso em ambiente, penso em tudo o que me rodeia. Animais plantas, paisagens e seres humanos" (E13). Essa concepção está, de acordo com uma estudante, associada a uma visão negativa do ambiente, ao afirmar que o que lhe vem à mente quando pensa em ambiente é "uma paisagem muito verde acompanhada por uma imagem de poluição. Pois infelizmente esta realidade [...] está muito presente nos nossos dias” (E31). Quanto às concepções que se focam na relação entre as pessoas e o mundo natural, a ideia de que as pessoas fazem parte do ambiente e são responsáveis por ele (Concepção 5) apresenta uma elevada frequência nas respostas das estudantes, cerca de 31 \%. Esta concepção ficou evidenciada em afirmações como:

\begin{abstract}
Ambiente é todo o que nos rodeia, até nas pequenas ações como o fumar, não ter cuidado com água e luz que são consumidas, assim como a reciclagem, que é uma ação que é bastante falada e que mesmo assim existe muitas pessoas que não a fazem. Por vezes são ações que pesamos só estar a prejudicar-nos mas que prejudicamos o próprio planeta e todos aqueles que nos rodeiam (E23).
\end{abstract}

Deve-se preservar os espaços verdes; fazer a reciclagem [...]. Promover o desenvolvimento sustentável, sendo exemplo para futuras gerações; Sermos ecológicos não deitando lixo para o chão, desligar as luzes, poupar água, etc. (E25).

A concepção de que o ambiente é o "conjunto de recursos naturais que tornam possível a sobrevivência humana e de todos os seres vivos" (URDA, 2004, inserir p.3) e, como salientam Loughland, Reid e Petocz (2002), contribui para a qualidade de vida das pessoas (Concepção 4) foi referida por algumas estudantes. Um ambiente não poluído surge associado ao "bem-estar" (E10) e a uma fonte de recursos de que o homem necessita para viver. À semelhança da Concepção 5, esta concepção descreve uma relação unidirecional entre os sistemas naturais e os sistemas sociais. Contudo, aqui as pessoas não se sentem parte integrante do ambiente e nem se preocupam com o "papel preponderante que o Homem assume na sua preservação" (E30), pretendendo apenas disfrutar daquilo que o ambiente lhes pode proporcionar. A única concepção que destaca uma relação bidirecional entre o mundo natural e o homem (Concepção 6) não foi evidenciada por nenhuma das estudantes.

\title{
4. Discussão e Conclusões
}

Nos últimos anos uma grande parte da investigação na área da educação ambiental tem-se debruçado sobre o estudo das concepções de ambiente, reconhecendo claramente a influência destas no processo de aprendizagem na educação formal (por exemplo, ALMEIDA, 2007; LOUGHLAND; REID; PETOCZ, 2002; TRACANA; FERREIRA; CARVALHO, 2012). As concepções dos alunos que revelam uma visão antropocêntrica do ambiente persistem até à idade adulta e são reveladoras do insucesso da educação ambiental implementada nas escolas (ALMEIDA; VASCONCELOS, 2013; LOUGHLAND; REID; PETOCZ, 2002; PETOCZ; REID; LOUGHLAND, 2003). Essa situação, de acordo com Shepardson et al. (2007), tem sido causada pelos currículos escolares, que apesar de enfatizarem uma abordagem interdisciplinar do ambiente, focam-se numa visão utilitarista dos recursos naturais, em que o ambiente sustenta a vida humana, em vez de uma visão ecocêntrica, que insere o homem no ambiente. Essa opinião é partilhada por Almeida e Vasconcelos (2013), exemplificando que a insistência em temáticas 
como a reciclagem nos projetos de escola só vem reforçar uma visão antropocêntrica do ambiente. Assim, não parece surpreendente que as estudantes que participaram neste estudo apresentem concepções de ambiente marcadamente antropocêntricas. Repare-se que nenhuma das participantes demonstrou uma concepção de ambiente que enfatizasse a relação bilateral entre os sistemas sociais e naturais, o que revela que existe, ainda, um longo caminho a percorrer no sentido de promover uma visão mais ecocêntrica do ambiente, condição essencial para a consecução dos objetivos da educação ambiental. Os profissionais de educação têm, naturalmente, um papel fundamental na educação ambiental; como tal, urge intervir na mudança de concepções no contexto da formação de professores. As concepções que esses profissionais possuem acerca do ambiente, muitas vezes, não se coadunam com os princípios da educação ambiental o que resulta em estratégias pedagógicas desadequadas e na perpetuação de concepções erróneas nos alunos. A este respeito, Flogaitis e Agelidou (2003) dão o exemplo de um estudo em que a maioria dos educadores de infância consideram os termos natureza e ambiente como sinónimos, o que também se verificou no estudo aqui reportado. No sentido de ultrapassar esses obstáculos alguns autores sugerem que devemos repensar a educação ambiental e trazê-la para fora do sistema formal de ensino, o que passa por uma intervenção junto das comunidades (LOUGHLAND et al., 2003) e na formação de profissionais que trabalhem diretamente com as populações, como é o caso dos educadores sociais e animadores socioculturais. A prioridade na educação ambiental, hoje, está em contribuir para uma melhor inclusão da educação ambiental nos currículos escolares, a par com um investimento ao nível da educação ambiental não formal.

\section{Referências}

ALERBY, E. A way of visualizing children's and young people's thoughts about the environment: A study of drawings. Environmental Education Research, London, v. 6, inserir n.3, p. 205-222. 2000.

ALMEIDA, A. Educaşão Ambiental. A importância da dimensão ética. Lisboa: Livros Horizonte, 2007.

ALMEIDA, A.; VASCONCELOS, C.M.; Teachers' Perspectives on the Human-Nature Relationship: Implications for Environmental Education. Research in Science

Education, v. 43, n. 1, p. 299-316, fev, 2013.

ALMEIDA, A.; VASCONCELOS, C.M.; STRECHT-RIBEIRO, O.; TORRES, J. Non-anthropocentric Reasoning in Children: Its incidence when they are confronted with ecological dilemmas. International Journal of Science Education, London, v. 35, n. 2, p. 312-334. 2013.

BAPTISTA, CM.; VILARIGUES, S. Futuro incerto. Educação ambiental em Portugal. Ozono, Madri, v. 12, p. 15-21. 2001.

BARRAZA, L. Children's drawings about the environment. Environmental Education Research, London, v. 5, n. 1, p. 49-67. 1999.

BJERKE, T.; KALTENBORN, B. The relationship of ecocentric and anthropocentric motives to attitudes toward large carnivores. Journal of Emironmental Psychology, Amsterdam, v. 19, n. 4, p. 415-421. 1999.

BOGDAN, R.; BIKLEN, S. Investigação qualitativa em educação: Uma introdução à teoria e aos métodos. Porto: Porto Editora, 1994.

DUNLAP, R.; VAN LIERE, K.; MERTIG, A.; JONES, R.E. Measuring endorsement of the New Ecological Paradigm: A revised NEP scale. Journal of Social Issues, Washington DC, v. 56, n. 3, p. 425-442. 2000. 
FLOGAITIS, E.; AGELIDOU, E. Kindergarten teachers' conceptions about nature and the environment. Environmental Education Research, London, v. 9, n. 4, p.461-478, dez. 2003.

GIORDAN, A. De la prise de conscience à l’action. Education Permanente, Ottawa, v. 148, n. 3, p. 19-29. 2001.

KAHN, P.; LOURENÇO, O. Water, air, fire, and earth: A developmental study in Portugal of environmental moral reasoning. Environment and Behaviour, v. 34, n. 4, p. 405-430. 2002.

KALVAITIS, D.; MONHARDT, R. The architecture of children's relationships with nature: A phenomenographic investigation seen through drawings and written narratives of elementary students. Environmental Education Research, London, v. 18, n. 2, p. 209-227. 2012.

KNAPP, D. The Thessaloniki Declaration: A wake-up call for environmental education? The Journal of Environmental Education, London, v. 31, n. 3, p. 32-39. 2000.

KILLINGSWORTH, M. J.; PALMER, J. Ecospeak: rhetoric and environmental politics in America. Carbondale, IL: Southern Illinois University Press, 1992.

LOCKWOOD, M. Humans valuing nature: synthesising insights from philosophy, psychology and economics. Environmental V alues, Isle Harris, UK, v. 8, n. 3, p. 381-401, ago. 1999.

LOUGHLAND, T.; REID, A.; PETOCZ, P. Young People's Conceptions of Environment: A phenomenographic analysis. Environmental Education Research, London, v. 8, n. 2, p. 187-197. 2002.

LOUGHLAND, T.; REID, A.; WALKER, K. PETOCZ, P. Factors influencing young people's conceptions of environment, Environmental Education Research, London, v. 9, n. 1, p. 3-20. 2003.

MÁXIMO-ESTEVES, L. Da teoria à prática: educação ambiental com as crianças pequenas ou o Fio da História. Porto: Porto Editora, 1998.

MERRIAM, S. Qualitative research. A guide to design and implementation. San Francisco, CA: Jossey Bass, 2009.

MILFONT, T. L.; DUCKITT, J. Preservation and utilization: understanding the structure of environmental attitudes. Medio Ambiente y Comportamiento Humano, México, v. 7, n. 1, p. 29-50. 2006.

OKSANEN, M. The moral value of biodiversity. Ambio, Estocolmo, v. 26, n. 8, p. 541-545, dez. 1997.

PALMER, J. Environmental education in the 21st century. Theory, practice progress and promise. London: Routledge Falmer, 1998.

PARLO, A.; BUTLER, M. Impediments to environmental education instruction in the classroom: A postworkshop inquiry. Journal of Environmental \& Science Education, London, v. 2, n. 1, p. 32-37. 2007.

PATTON, M.Q. Qualitative evaluation and research methods. 3ed. Newbury Park, CA: Sage Publications, 2002.

PETOCZ, P.; REID, A.; LOUGHLAND, T.. 'The importance of adults' conceptions of the environment for education. AUSTRALIAN ASSOCIATION FOR RESEARCH IN EDUCATION CONFERENCE, Auckland, 2003. Proceedings... Auckland: University of Auckland, 2003. Disponível em: <www.aare.edu.au/index.htm>. Acesso em: 7 dez. 2010.

SHEPARDSON, D. Student ideas: What is an environment? The Journal of Environmental Education, London, v. 36, n. 4, p. 49-58. 2005. 
SHEPARDSON, D.; WEE, B.; PRIDDY, M.; HARBOR, J. Students' mental models of the environment. Journal of Research in Science Teaching, New York, v. 44, n. 2, p. 327-348, fev. 2007.

SINGER, P. Animal liberation. London: Jonathan Cape, 1990.

STEM, P.C.; DIETZ, T.; KALOF, L. Value orientations, gender, and environmental concern, Environment and Behavior, v. 25, n. 5, p. 322-348. 1993.

STRAUSS, A.; CORBIN, J. Basic of qualitative research. Techniques and procedures for developing grounded theory. Thousand Oaks, CA: Sage Publications, 1998.

TAYLOR, P. Respect for nature. A theory of environmental ethics. Princeton: Princeton University Press, 1989.

THOMPSON, S.G.; BARTON, M. Ecocentric and anthropocentric attitudes toward the environment. Journal of Environmental Psychology, Amsterdam, v. 14, n. 2, p. 149-157, jun. 1994.

TRACANA, R.B.; FERREIRA, M.E.; CARVALHO, G. Concepções de (futuros) professores portugueses sobre educação ambiental: Identificação das dimensões 'ecocêntrica', 'antropocêntrica' e 'sentimentocêntrica'. Revista Brasileira de Pesquisa em Educação em Ciências, São Paulo, v. 12, n. 2, p. 111-127. 2012.

UNITED NATIONS EDUCATIONAL, SCIENTIFIC AND CULTURAL ORGANIZATION / UNITED NATIONS ENVIRONMENT PROGRAMME (UNESCO-UNEP). The Belgrade charter: A global framework for environmental education. Connect, Washington DC, v. 1, n. 1, p. 1-9. 1976.

URDA, E.G. Las concepciones del medioambiente en estudiantes del nivel superior. Revista Iberoamericana de Educación, Madrid, v.2, n.2, p.1-7. 2004. p. 1-7. Disponível em:

<www.rieoei.org/deloslectores/602Gonzalez.PDF>. Acesso em: 3 set. 2009.

WALSHE, N. Understanding students' conceptions of sustainability. Environmental Education Research, London, v. 14, n. 5, p. 537-558. 2008.

WISEMAN, M.; BOGNER F. A higher-order model of ecological values and its relationship to personality. Personality and Individual Differences, Utah, v. 34, n. 5, p. 783-794, abr. 2003.

YIN, Robert. Case study research: Design and methods. 3ed. Newbury Park, CA: Sage Publications, 2003.

Artigo submetido em 09/05/2013

Artigo aprovado em 18/09/2013 